\section{WHO congress backs traditional medicine}

The World Health Organization (WHO) has endorsed an international agreement that promises to give traditional medicines a foothold in health systems around the world.

At its first Congress on Traditional Medicine in Beijing on 8 November, the WHO unveiled the 'Beijing Declaration'. The declaration calls on member states of the United Nations to formulate policies for ensuring the safe and effective use of traditional medicines, to create systems for licensing practitioners, and to encourage communication between

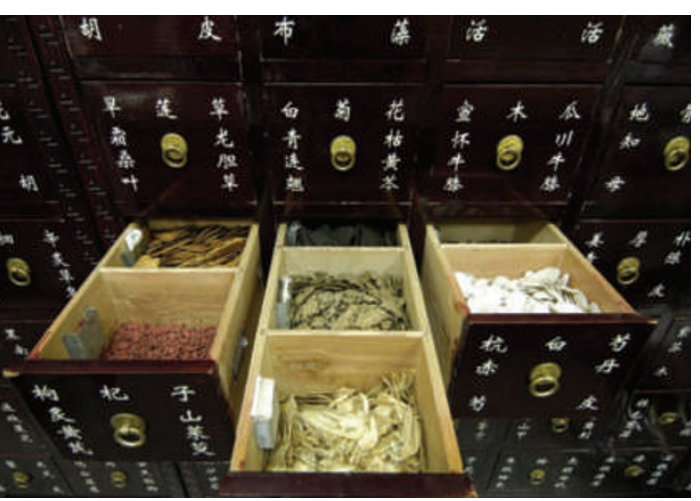

Chinese medicine is coming in from the cold.
Western and traditional practitioners.

China in particular has long been keen on the international acceptance of traditional medicines. But researchers have had difficulty pinning down and standardizing the active ingredients, as these are often found in plant extracts that have regional or seasonal variations. Because of this, drug regulatory agencies around the world have hesitated to approve their use.

\section{Proposed reform to animal testing rules draws fire}

The European Commission last week unveiled a proposal to reform the regulation of scientific experiments that use animals.

The commission's suggested update to the 20-year-old 86/609/EEC directive will now be considered by the European Parliament, probably in 2009. However, business and academic groups are pushing for a number of changes, voicing concern over the draft's proposals to limit the reuse of the same animal in a series of experiments, and to curb the use of specimens captured from the wild.

Primate researchers are particularly worried by elements of the proposed directive that could prevent any basic research on non-human primates that does not relate to either the survival of the species or to serious clinical conditions in humans. All experiments on great apes would be banned, except during extreme circumstances such as pandemics.

\section{Contaminants identified in plastic lab equipment}

Thousands of scientists could be unwittingly ruining their experiments merely by using standard plastic lab equipment, according to a study published in Science. Andrew Holt of the University of Alberta in Edmonton, Canada, found that a disinfectant and a lubricant used in the manufacture of plastic pipette tips, tubes and micro-plates could leach into common solvents, including water (G. R. McDonald et al. Science 322, 917; 2008).

Holt and his team determined that the contaminants are potent enzyme inhibitors, and skewed their drug-activity assays. "People in any big science department will say that they don't trust plastic in some experiments," says Holt. But the compounds responsible had never been identified before, he adds. Eppendorf, a manufacturer of some of the plastic products, says that "so far, we have not experienced any product problems with our customers due to these substances". For a longer version of this story, see http://tinyurl.com/5z3hau. 